\title{
Automedicação entre estudantes de enfermagem e medicina no Brasil: revisão integrativa
}

\author{
Self-medication among nursing and medical students in Brazil: integrative review \\ Automedicación entre estudiantes de enfermería y medicina en Brasil: revisión integrativa
}

Recebido: 10/03/2021 | Revisado: 17/03/2021 | Aceito: 03/02/2022 | Publicado: 05/02/2022

Thaís Martins dos Santos

ORCID: https://orcid.org/0000-0002-4957-9995 Universidade do Estado de Mato Grosso, Brasil

E-mail: thais.martins@unemat.br

Taís Aparecida Zattar

ORCID: https://orcid.org/0000-0002-6827-2286 Universidade do Estado de Mato Grosso, Brasil

E-mail: taiszattar24@hotmail.com

Bianca Teshima de Alencar

ORCID: https://orcid.org/0000-0001-6812-3494 Universidade do Estado de Mato Grosso, Brasil

E-mail: bianca.teshima@unemat.br

Mariana Lenina Menezes Aleixo

ORCID: https://orcid.org/0000-0002-9363-2423 Universidade do Estado de Mato Grosso, Brasil E-mail: mariana.aleixo@unemat.br

Bárbara Maria Santana Costa ORCID: https://orcid.org/0000-0003-0269-0124 Universidade do Estado de Mato Grosso, Brasil

E-mail: barbaramariascosta@gmail.com

Larissa Maria Scalon Lemos

ORCID: https://orcid.org/0000-0003-2706-0305 Universidade do Estado de Mato Grosso, Brasil E-mail:1mscalon@hotmail.com

\begin{abstract}
Resumo
A automedicação é considerada um problema de saúde no Brasil e no mundo. Vários os fatores facilitam essa prática, incluindo dificuldade no acesso a médicos e facilidade no acesso a medicamentos, propaganda, internet e conhecimento sobre medicamentos e doença. Embora a automedicação tenha vantagens, os problemas decorrentes da sua prática são mais preocupantes. Este estudo teve como objetivo realizar uma revisão integrativa sobre o panorama da automedicação entre estudantes de enfermagem e medicina no Brasil. O levantamento e seleção de material foi realizado pela Biblioteca Virtual as Saúde nas bases de dados da Medline, Lilacs e BDENF, entre setembro de 2020 a janeiro de 2021. Para as buscas controladas utilizou-se os DeCS automedicação, estudantes de enfermagem e estudantes de medicina. Foram obtidos 15 artigos, dentre eles, 53,3\% foram realizados com estudantes do curso de medicina, 26,6\% de enfermagem e $20 \%$ com alunos de ambos os cursos. A maioria das publicações é de 2019 (26,6\%), $40 \%$ são provenientes do Estado de São Paulo e 60\% estão indexadas na base de dados da Lilacs. Constatouse que o uso de analgésicos foi prevalente em $86,6 \%$ dos estudos, e que estudantes do sexo feminino praticam mais a automedicação. A automedicação não é uma prática que deva ser eliminada, mas utilizada com cautela e orientações corretas, entretanto, é necessário modificar os paradigmas na formação acadêmica para a formação de profissionais mais qualificados visando a segurança do paciente.
\end{abstract}

Palavras-chave: Farmacologia; Ciências da saúde; Analgésicos; Prescrições.

\begin{abstract}
Self-medication is considered a health problem in Brazil and worldwide. Several factors allow such practice, for instance difficulty in accessing doctors and easy access to medicines, marketing, internet and knowledge about the illness and the medicines. Although self-medication has advantages, the problems arising from its practice are more worrying. This study aimed to conduct an integrative review on the panorama of self-medication among nursing and medical students in Brazil. The databases Medline, Lilacs and BDENF in the Biblioteca Virtual as Saúde were scrutinized using "automedicação", "estudantes de enfermagem" and "estudantes de medicina" key words, between September 2020 and January 2021. Fifteen articles were obtained from wich 53.3\% including medical students, $26.6 \%$ nursing students and 20\% students from both courses. Most publications are from 2019 (26.6\%), from the state of Sâo Paulo (40\%), and 60\% are Lilacs database indexed. The use of analgesics as self-medication was prevalent in $86.6 \%$
\end{abstract}


of the included studies, and that female students are more related to this practice. Self-medication is not a practice that should be eliminated, but used with caution, following correct guidelines, however, it is necessary to change paradigms in academic training to create more qualified professionals aiming at patient safety.

Keywords: Pharmacology; Health sciences; Analgesics; Prescription.

\section{Resumen}

La automedicación es considerada un problema de salud en Brasil y en el mundo. Varios factores facilitan esta práctica, incluyendo la dificultad de acceso a los médicos y la facilidad de acceso a medicamentos, publicidad, Internet y conocimientos sobre medicamentos y enfermedades. Aunque la automedicación tiene ventajas, los problemas derivados de su práctica son más preocupantes. Este estudio tuvo como objetivo realizar una revisión integrativa sobre el panorama de la automedicación entre estudiantes de enfermería y medicina en Brasil. La recolección y selección de material fue realizada por la Biblioteca Virtual as Saúde en las bases de datos de Medline, Lilacs y BDENF, entre septiembre de 2020 a enero de 2021. Para las búsquedas controladas se utilizaron los DeCS automedicación, estudiantes de enfermería y estudiantes de medicina. Fueron obtenido 15 artículos, entre ellos, 53,3\% fueron realizados con estudiantes del curso de medicina, $26,6 \%$ de enfermería y $20 \%$ con alumnos de ambos cursos. La mayoría de las publicaciones son de 2019 (26,6\%), 40\% son provenientes del Estado de São Paulo y 60\% están indexadas en la base de datos de Lilacs. Se constató que el uso de analgésicos fue prevalente en el 86,6\% de los estudios, y que estudiantes del sexo femenino practican más la automedicación. La automedicación no es una práctica que deba ser eliminada, pero utilizada con precaución y orientaciones correctas, sin embargo, es necesario modificar los paradigmas en la formación académica para la formación de profesionales más calificados visando la seguridad del paciente.

Palabras clave: Farmacología; Ciencias de la salud; Analgésicos; Recetas.

\section{Introdução}

O uso dos medicamentos acompanha a história da evolução humana, desde os primórdios da civilização humana, sendo utilizada com a finalidade de alento, alivio e cura de enfermidades. Com avanço das eras e melhora das tecnologias e meios de pesquisa, passa a ser cada vez mais utilizada e difundida pelo mundo todo. Porem alinhado a isto surge à problemática do uso não racional dos medicamentos por parte da população de forma indiscriminada e sem prescrição (Silva \& Alvin, 2020).

A Organização Mundial da Saúde (OMS) e a Agencia Nacional de Vigilância Sanitária (ANVISA), definem automedicação como sendo "o uso de medicamento sem a prescrição, orientação e ou supervisão de um médico ou dentista" (OMS, 1998; Brasil, 2001).

Embora automedicação possa ser vista como uma solução rápida para o alívio de sintomas, sendo essa uma de suas vantagens, as desvantagens da prática são causas de preocupação. Um medicamento adquirido sem prescrição pode ser usado na posologia e esquema de tratamento escolhidas pelo indivíduo, podendo ir de uma subdose sem efeito até uma dose excessiva com efeitos tóxicos. Outro fator relevante é a interação. Estudos demonstram que a associação de dois fármacos aumenta a probabilidade de Reações Adversas ao Medicamento (RAM) e que o uso de número superior a cinco medicamentos eleva o risco do acometimento por eventos adversos relacionados a Interações Medicamentosas (IM) (Melo \& Pauferro, 2020).

A prática de automedicação é um evento global (Arrais et al., 2016), e no Brasil tal pratica é exercida por cerca de 80 milhões de pessoas, sendo que 35\% dos medicamentos adquiridos são para automedicação e destes 44,1\% necessitam de prescrição para sua aquisição. O Brasil destaca-se como o primeiro país da América Latina e pela quinta posição no ranking mundial no consumo de medicamentos, acarretando em cerca de 24 mil óbitos anuais relacionados à intoxicação medicamentosa no país (Mota et al., 2008; Bispo et., 2020).

Um grande passo para o reconhecimento que os benefícios dos medicamentos podem não se concretizar ou até mesmo prejudicar a saúde do usuário se não forem usados de forma adequada, ocorre a partir da Conferencia de Nairobi (Bermudez et al., 2018). No entanto, mesmo antes disso, a dimensão do problema fez com que, no Brasil, em 1988, o Ministério da Saúde lançasse a Política Nacional de Medicamentos por meio da Portaria no 3916 de outubro de 1998, afim de 
desenvolver estratégias para promover do uso racional de medicamentos (Brasil, 1998), e em 2013, a Portaria n ${ }^{\circ} 834$ de 14 de Maio de 2013, com a finalidade de "estabelecer, coordenar e monitorar os sistemas de vigilância toxicológica e farmacológica, além de, respeitada a legislação em vigor, regulamentar, controlar e fiscalizar os produtos e serviços que envolvam risco à saúde pública", a partir da qual ficam definidas estratégias para Promoção do Uso Racional de Medicamentos (URM) no âmbito do Ministério da Saúde (Brasil, 2013).

São vários os fatores que propiciam a automedicação no Brasil. A ANVISA (2020) aponta os Medicamentos Isentos de Prescrição (MIP) como grandes colaboradores dessa prática. Embora eles sejam isentos de prescrição médica, eles não são isentos de uso com orientação já que, como qualquer medicamento, apresentam efeitos colaterais e riscos de interações medicamentosas. No geral, os MIP's corresponderam a 65,5\% do total de medicamentos usados para automedicação. Macedo et al. (2016) elencaram a propaganda e marketing como outro fator de grande importância no uso irracional de medicamentos. Utilizam da persuasão e incentivo no consumo de medicamentos, mesmo que para atingir esse objetivo ocorra a criação de uma nova necessidade no consumidor.

Estudos demonstram que a busca por automedicação está relacionada a vários fatores, entre eles, o conhecimento próprio sobre o medicamento, indicação de amigos e familiares, dificuldade e demora em se conseguir uma consulta pelo SUS, falta de atenção do profissional médico quando a consulta acontece, e ainda pelo fato atendentes de farmácia, que na maioria das vezes não é o profissional farmacêutico, tenta vender medicamentos para que possa incrementar seus ganhos. Muitos medicamentos que, pela lei brasileira, deveriam ser vendidos apenas mediante apresentação da receita, são dispensados livremente (Naves et al., 2008; Bispo et al., 2017).

O padrão de automedicação varia substancialmente entre diferentes populações e seus fatores (idade, gênero, aspectos sócio-econômico-culturais, nível educacional, gravidade da doença, entre outros) (Andrade et al., 2020). Essa prática atinge indiscriminadamente todas as classes sociais, no entanto, estudos têm demonstrado uma maior ocorrência entre aqueles que possuem maior grau de informação. $\mathrm{O}$ acúmulo de informação relacionada ao uso de medicamentos parece conferir maior confiança na prática a medicação (Cavalgante \& Khouri, 2019). Referente a este fato, estudos vêm demonstrando uma maior prevalência de automedicação entre os estudantes universitários, especialmente em cursos da área da saúde (Domingues et al., 2017).

Automedicação entre estudantes universitários vem crescendo e se tornando uma problemática no mundo todo. Em uma revisão de meta-análise com estudos de diversos países, incluindo o Brasil mostraram uma prevalência geral de automedicação entre estudantes universitários de 70,1\% (95\% CI: 64,3\%-75,4\%), sendo que entre estes 97,2\% estudantes eram da área médica, e 44,7\%, de outras áreas do conhecimento, apontando para uma relação com o fato dos estudantes das áreas da saúde possuírem maior conhecimento sobre as drogas (Behzadifar et al., 2020).

Embora os estudos relacionados a pratica de automedicação em estudantes universitários seja atual e crescente, a literatura sobre essa prática entre estudantes de Medicina e Enfermagem no Brasil ainda é incipiente, com estudos pontuais, fato que motivou a realização desta revisão no intuito de se obter uma visão geral do panorama da automedicação entre estudantes desses cursos no Brasil.

\section{Metodologia}

Foi realizada uma revisão integrativa de literatura, norteada pela seguinte questão problema: a) Como a prática da automedicação ocorre entre estudantes dos cursos de Enfermagem e Medicina no Brasil?

Para Botelho et al. (2011), o método da revisão integrativa viabiliza a capacidade de sistematização do conhecimento científico de forma que o pesquisador se aproxime da problemática que deseja apreciar. Sendo assim, é 
possível traçar um panorama sobre sua produção científica para conhecer a evolução do tema ao longo do tempo e, com isso, visualizar possíveis oportunidades de pesquisa. Este foi o objetivo dos autores ao realizar a presente revisão.

Este trabalho seguiu seis passos da revisão, conforme recomenda o Centro Cochrane no Brasil, independentemente de sua especificidade, sendo eles: 1) formulação da pergunta; 2) localização dos estudos; 3) avaliação crítica dos estudos; 4) coleta de dados; 5) análise e apresentação dos dados; 6) interpretação dos dados (Botelho et al., 2011).

O levantamento bibliográfico foi realizado entre setembro de 2020 e janeiro de 2021, a partir das bases de dados eletrônicas em saúde internacionais e nacionais indexadas no portal da Biblioteca Virtual de Saúde (BVS): Medical Literature Analysis and Retrieval System Online (Medline), Literatura Latino-Americana e do Caribe em Ciências da Saúde (Lilacs) e na base de dados bibliográficos especializada na área de Enfermagem (BDENF).

Os termos utilizados para a realização das buscas foram os Descritores em Ciências da Saúde (DeCS): automedicação; "estudantes de enfermagem"; "estudantes de medicina”, "profissionais da saúde". O cruzamento dos descritores foi feito de forma controlada a partir dos operadores booleanos and e and not. A expressão de busca aplicada para a identificação e seleção dos materiais está apresentada na figura 1.

Quanto aos critérios de seleção, foram adotados para a inclusão de estudos: artigos completos que abordassem somente a automedicação em estudantes dos cursos de medicina e enfermagem; publicados no período de 2010 a 2020; no idioma português; indexados nas bases de dados da Medline, Lilacs e Bdenf. Foram excluídos artigos duplicados, artigos de revisão, bem como aqueles que após a leitura do título e resumo não responderam ao foco do estudo. Após aplicar esses critérios, a amostra final compreendeu 15 artigos lidos na íntegra e analisados com base no objetivo proposto.

Os procedimentos utilizados para a busca e seleção dos artigos na BVS como a expressão de busca controlada, os critérios de inclusão e exclusão constam apresentados na figura 1, como recomendado pelo Preferred Reporting Items for Systematic Reviews and Meta-Analyses (Prisma), conforme descrito por Moher et al. (2015).

Para a análise de dados, foi elaborado um instrumento para a categorização dos principais dados, possibilitantando o cálculo em percentual (\%) com os seguintes itens: título do artigo, autores, ano de publicação, base de dados, periódico, local do estudo, método (tipo de estudo e amostra), principais resultados (classe de fármacos mais utilizadas, sexo, período do curso).

Por último, a análise crítica e síntese dos manuscritos que compuseram a pesquisa foram apresentadas em duas categorias: a) perfil das produções sobre o tema; b) principais resultados relacionados a automedicação entre os estudantes de enfermagem e medicina.

\section{Resultados e discussão}

Na Tabela 1, estão apresentadas as variáveis do perfil das produções selecionadas: autores, ano de publicação, periódico, local do estudo e método. Dentre os 15 artigos analisados, 53,3\% foram realizados com estudantes do curso de medicina, 26,6\% de enfermagem e $20 \%$ com alunos de ambos os cursos. Quanto ao método, 100\% são de delineamento transversal, conforme apresentado na Tabela 1. 
Figura 1 - Fluxograma do procedimento de busca e seleção dos artigos incluídos na amostra, conforme Prisma (Cáceres- MT, 2021).

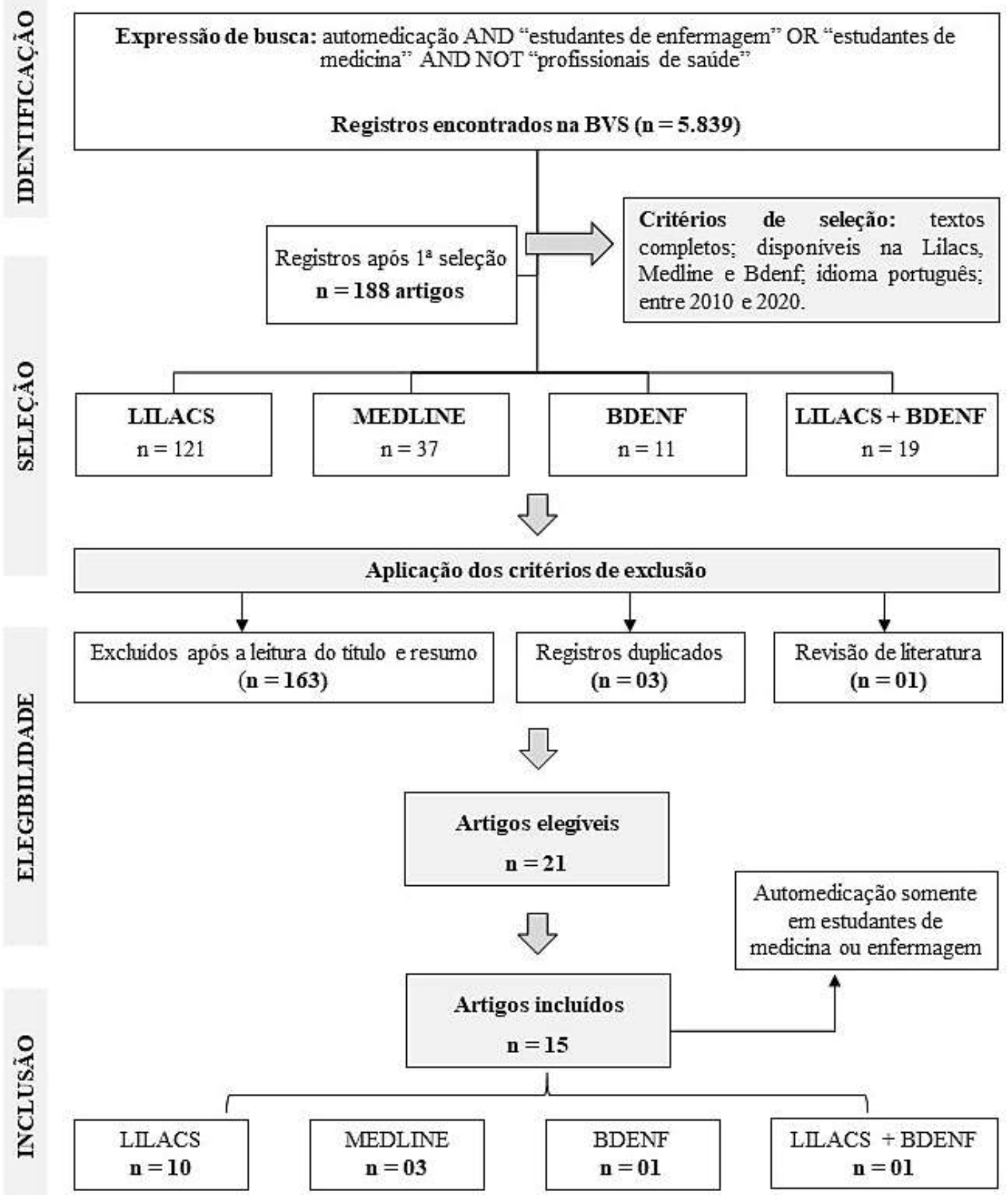

Fonte: Autores. 
Tabela 1 - Perfil das produções selecionadas para análise segundo autores, ano de publicação, periódico, Qualis capes (QC), local do estudo método. (Cáceres-MT, 2021).

\begin{tabular}{|c|c|c|c|c|c|}
\hline Id. & Autor e ano & Base de dados & Periódico & Local do estudo & $\begin{array}{c}\text { Método } \\
\text { (estudo/amostra) } \\
\end{array}$ \\
\hline A1 & $\begin{array}{l}\text { Moraes et al. } \\
2018\end{array}$ & Lilacs & $\begin{array}{c}\text { Revista da Sociedade } \\
\text { Brasileira de Clínica Médica }\end{array}$ & $\begin{array}{l}\text { Espirito Santo } \\
\text { (ES) }\end{array}$ & $\begin{array}{l}\text { Transversal, quantitativo } \\
148 \text { est. de medicina }\end{array}$ \\
\hline A2 & $\begin{array}{l}\text { Santos et al. } \\
2019\end{array}$ & Lilacs & $\begin{array}{c}\text { Revista brasileira de } \\
\text { neurologia }\end{array}$ & Alagoas (AL) & $\begin{array}{l}\text { Transversal, quantitativo } \\
97 \text { est. de medicina }\end{array}$ \\
\hline $\mathbf{A 3}$ & $\begin{array}{c}\text { Tognoli et al. } \\
2019\end{array}$ & Lilacs & $\begin{array}{l}\text { Journal of Health \& } \\
\text { Biological Sciences }\end{array}$ & Fernandópolis (SP) & $\begin{array}{l}\text { Transversal, quantitativo } \\
320 \text { est. de medicina }\end{array}$ \\
\hline A4 & Alves et al. 2019 & Bdenf & $\begin{array}{c}\text { Revista de enfermagem } \\
\text { UFPE on line }\end{array}$ & João Pessoa (PB) & $\begin{array}{l}\text { Transversal, quantitativo } \\
100 \text { est. de enfermagem }\end{array}$ \\
\hline A5 & $\begin{array}{l}\text { Carneiro et al. } \\
2019\end{array}$ & Lilacs & $\begin{array}{l}\text { Revista de medicina (São } \\
\text { Paulo) }\end{array}$ & Sobral (CE) & $\begin{array}{l}\text { Transversal, quantitativo } \\
219 \text { est. de medicina }\end{array}$ \\
\hline A6 & $\begin{array}{c}\text { Gama \& Secoli } \\
2017 \\
\end{array}$ & Medline & $\begin{array}{c}\text { Revista gaúcha de } \\
\text { enfermagem }\end{array}$ & Coari (AM) & $\begin{array}{l}\text { Transversal, quantitativo } \\
116 \text { est. de enfermagem }\end{array}$ \\
\hline A7 & $\begin{array}{c}\text { Pilger et al. } \\
2016\end{array}$ & Lilacs & Revista AMRIGS & Pelotas (RS) & $\begin{array}{l}\text { Transversal, quantitativo } \\
609 \text { est. de medicina }\end{array}$ \\
\hline A8 & Silva et al. 2014 & Lilacs e Bdenf & $\begin{array}{l}\text { Revista eletrônica de } \\
\text { enfermagem }\end{array}$ & São Paulo & $\begin{array}{c}\text { Coorte transversal } \\
283 \text { est. de medicina e } \\
\text { enfermagem }\end{array}$ \\
\hline A9 & Martinez et al. 2014 & Lilacs & $\begin{array}{c}\text { Revista brasileira de } \\
\text { Reumatologia }\end{array}$ & Sorocaba (SP) & $\begin{array}{c}\text { Transversal, quantitativo } \\
83 \text { est. medicina e enfermagem }\end{array}$ \\
\hline A10 & Zubaran \& Lazzaretti 2013 & Medline & Einstein (São Paulo) & Santa Catarina (RS) & $\begin{array}{c}\text { Transversal, quantitativo } \\
342 \text { est. de enfermagem e } \\
\text { medicina }\end{array}$ \\
\hline A11 & Santos et al. 2012 & Lilacs & $\begin{array}{l}\text { Journal of the Health } \\
\text { Sciences Institute }\end{array}$ & São Paulo & $\begin{array}{l}\text { Transversal/quantitativo } \\
89 \text { est. de enfermagem }\end{array}$ \\
\hline A12 & Silva et al. 2012 & Lilacs & $\begin{array}{l}\text { Medicina (Ribeirão Preto. } \\
\text { Impresso) }\end{array}$ & São Paulo & $\begin{array}{c}\text { Transversal/quantitativo } \\
200 \text { est. de medicina }\end{array}$ \\
\hline A13 & Trevisol et al. 2011 & Lilacs & $\begin{array}{c}\text { Revista da Sociedade } \\
\text { Brasileira de Clínica Médica }\end{array}$ & Tubarão (SC) & $\begin{array}{c}\text { Transversal/quantitativo } \\
80 \text { est. de medicina }\end{array}$ \\
\hline A14 & $\begin{array}{l}\text { Souza et al. } \\
2011\end{array}$ & Medline & $\begin{array}{c}\text { Revista latino-americana de } \\
\text { enfermagem }\end{array}$ & Goiânia (GO) & $\begin{array}{l}\text { Transversal/quantitativo } \\
250 \text { est. de enfermagem }\end{array}$ \\
\hline A15 & Fonseca et al. 2010 & Lilacs & Diagnóstico e tratamento & Santos (SP) & $\begin{array}{c}\text { Transversal/quantitativo } \\
299 \text { est. de medicina }\end{array}$ \\
\hline
\end{tabular}

Fonte: Autores (2021).

O ano de maior destaque em quantidade de publicações foi 2019 com 26,6\%. Entretanto, analisando as publicações em períodos de cinco anos, observa-se que 46,6\% são referentes ao período de 2015 à 2020 e 53,33\% de 2015 à 2010 . Em relação ao local de coleta e realização dos estudos, constatou-se que 40\% deles é do Estado de São Paulo. A maioria dos artigos (60\%) são produções de revistas indexadas na base de dados da Lilacs (Tabela 1).

Nos estudos selecionados, 33,3\% dos autores incluíram alunos de medicina e de enfermagem do $1^{\circ}$ ao $4^{\circ}$ ano de graduação. Os autores Santos et al. (2019) e Tognoli et al. (2019), justificam essa escolha pela dificuldade de contato com turmas do último ano em virtude de carga horária cumprida em plantões, centro cirúrgico, unidades básicas de saúde ou prontossocorros.

Na Tabela 2 estão reunidas informações sobre a prática da automedicação entre os estudantes de enfermagem e medicina, mostrando a grande frequência desta prática entre esses alunos. Dos estudos selecionados, 86,6\% acusaram que o uso de analgésicos é predominante na automedicação, comparado às demais classes medicamentosas. Além dos analgésicos, compõem essa lista os antitérmicos, antiácidos e antimicrobianos, bem como o uso de psicofármacos e moderadores de apetite. Gama e Secoli (2017), identificaram que dentre os medicamentos, os mais consumidos via automedicação são o paracetamol e 
dipirona (48,8\%), cefalexina $(6,0 \%)$ e complexo B $(8,3 \%)$. Entre os antimicrobianos, os mais utilizados foram cefalexina $(55,6 \%)$, amoxicilina $(22,2 \%)$, ampicilina $(11,1 \%)$ e azitromicina $(11,1 \%)$.

Tabela 2 - Informações sobre a prática da automedicação entre estudantes de enfermagem e medicina do Brasil, nos artigos levantados segundo os critérios de inclusão (Cáceres-MT, 2021).

\begin{tabular}{|c|c|c|c|c|}
\hline Id. & $\begin{array}{l}\text { Curso/ } \\
\text { período }\end{array}$ & Classe de fármacos mais usada & $\begin{array}{l}\text { Prevalência da } \\
\text { automedicação }\end{array}$ & Motivos da automedicação \\
\hline A1 & $\begin{array}{l}\text { Medicina } \\
1^{\circ} \text { ao } 4^{\circ} \text { ano }\end{array}$ & $\begin{array}{l}\text { Analgésicos, Anti-inflamatórios, Antiácidos, } \\
\text { psicotrópicos }\end{array}$ & $\begin{array}{l}1^{\circ} \text { e } 2^{\circ}=44,57 \% \\
3^{\circ} \text { e } 4^{\circ}=71,42 \%\end{array}$ & $\begin{array}{l}\text { Dor, febre/inflamação, azia/indigestão, } \\
\text { insônia, falta de concentração para os } \\
\text { estudos, e sobrepeso }\end{array}$ \\
\hline A2 & $\begin{array}{c}\text { Medicina } \\
1^{\circ} \text { ao } 6^{\circ} \text { ano } \\
\end{array}$ & Analgésico comuns & $76,5 \%$ & Estresse \\
\hline A3 & $\begin{array}{c}\text { Medicina } \\
1^{\circ} \text { ao } 4^{\circ} \text { ano }\end{array}$ & $\begin{array}{l}\text { Analgésicos, Anti-inflamatórios, Antigripais, } \\
\text { Relaxantes musculares, antitérmicos }\end{array}$ & $96,56 \%$ & $\begin{array}{l}\text { Cefaleia, mialgia, resfriado comum, } \\
\text { epigastralgia e febre }\end{array}$ \\
\hline A4 & $\begin{array}{l}\text { Enfermagem } \\
1^{\circ} \text { ao } 5^{\circ} \text { ano }\end{array}$ & $\begin{array}{l}\text { Analgésicos/Antitérmicos, } \\
\text { inflamatórios, Antigripais, Xaropes para } \\
\text { tosse, Complexos } \\
\begin{array}{l}\text { Descongestionantes nasais, Antánicos, } \\
\text { antialérgicos }\end{array}\end{array}$ & $99,0 \%$ & Dor e febre \\
\hline A5 & $\begin{array}{c}\text { Medicina } \\
1^{\circ} \text { ao } 4^{\circ} \text { ano }\end{array}$ & Analgésicos & $\begin{array}{l}72,2 \% \text { - Cefaleia } \\
87,2 \% \text { - Enxaqueca }\end{array}$ & $\begin{array}{l}\text { Pré-provas, ansiedade, estresse, } 4 \text { horas } \\
\text { ou menos de sono }\end{array}$ \\
\hline A6 & $\begin{array}{l}\text { Enfermagem } \\
1^{\circ}, 3^{\circ}, 5^{\circ}, 7^{\circ} \mathrm{e} \\
9^{\circ} \text { semestre }\end{array}$ & $\begin{array}{l}\text { Anti-inflamatórios (AINES), polivitamínicos, } \\
\text { anti-histamínicos, antimicrobianos } \\
\text { Anticoncepcionais }\end{array}$ & $76,0 \%$ & $\begin{array}{l}\text { Dor - dores de cabeça, abdominais e } \\
\text { cólicas menstruais, infecções de } \\
\text { garganta e urinária, resfriado, febre, } \\
\text { problemas gastrintestinais, } \\
\text { contracepção, presença de alergias, } \\
\text { diarreia e tosse }\end{array}$ \\
\hline A7 & $\begin{array}{c}\text { Medicina } \\
1^{\circ} \text { ao } 4^{\circ} \text { ano }\end{array}$ & $\begin{array}{l}\text { Analgésicos, Anti-inflamatórios (AINES), } \\
\text { Antitérmicos, Antibióticos, Psicofármacos }\end{array}$ & $90 \%$ & Febre, cefaleia e mialgia \\
\hline A8 & $\begin{array}{l}\text { Enfermagem } \\
1^{\circ} \text { e } 4^{\circ} \\
\text { semestres }\end{array}$ & $\begin{array}{l}\text { Analgésicos, antitérmicos, Anti- } \\
\text { inflamatórios, Antialérgicos, Antibióticos }\end{array}$ & $\begin{array}{l}1^{\circ} \text { semestre }-86,5 \% \\
4^{\circ} \text { semestre }-100 \%\end{array}$ & $\begin{array}{l}\text { Falta de tempo, alivio da dor, mesmo } \\
\text { problema, demora no agendamento de } \\
\text { consulta médica, possui conhecimento } \\
\text { científicos, facilidade de acesso. }\end{array}$ \\
\hline A9 & $\begin{array}{l}\text { Enfermagem e } \\
\text { medicina } \\
\text { Não } \\
\text { especificado } \\
\end{array}$ & $\begin{array}{l}\text { Analgésicos, anti-inflamatórios, opioides, } \\
\text { antidepressivos }\end{array}$ & $63,8 \%$ & Alívio da dor \\
\hline A10 & $\begin{array}{l}\text { Enfermagem e } \\
\text { medicina } \\
\text { Não } \\
\text { especificado }\end{array}$ & $\begin{array}{l}\text { Moderadores de apetite, (anfepramona e } \\
\text { femproporex) }\end{array}$ & $\begin{array}{l}26,66 \% \text { apenas na } \\
\text { enfermagem }\end{array}$ & Perda de peso \\
\hline A11 & $\begin{array}{l}\text { Enfermagem } \\
8^{\circ} \text { semestre }\end{array}$ & $\begin{array}{l}\text { Analgésicos, anti-inflamatórios, antitérmicos, } \\
\text { antibióticos, anticoncepcionais }\end{array}$ & $65,17 \%$ & $\begin{array}{l}\text { Conhecimento da patologia, ação da } \\
\text { droga e alívio rápido dos sintomas } \\
\text { apresentados, falta de tempo, } \\
\text { dificuldade em conseguir consulta, não } \\
\text { ter convênio médico, necessidade de } \\
\text { permanecer acordado }\end{array}$ \\
\hline A12 & $\begin{array}{c}\text { Medicina } \\
\text { Todos os anos } \\
\end{array}$ & $\begin{array}{l}\text { Analgésicos, antitérmicos, antigripais, } \\
\text { descongestionantes nasais, antibióticos }\end{array}$ & $92 \%$ & Não especificado \\
\hline A13 & $\begin{array}{l}\text { Medicina } \\
\quad \text { Não } \\
\text { especificado } \\
\end{array}$ & Analgésicos e antitérmicos & $81,2 \%$ & Alívio da dor e febre \\
\hline A14 & $\begin{array}{l}\text { Enfermagem } \\
\quad \text { Não } \\
\text { especificado }\end{array}$ & $\begin{array}{l}\text { Analgésicos (dipirona, paracetamol), anti- } \\
\text { inflamatórios não esteroidais (ácido } \\
\text { acetilsalicílico). }\end{array}$ & Situações de dor $38,8 \%$ & $\begin{array}{l}\text { Falta de tempo para ir ao médico, os } \\
\text { próprios conhecimentos ajudam na } \\
\text { escolha do medicamento e o acesso ao } \\
\text { balconista da farmácia é mais rápido e } \\
\text { barato }\end{array}$ \\
\hline A15 & $\begin{array}{c}\text { Medicina } \\
\text { Todos os anos }\end{array}$ & $\begin{array}{l}\text { Analgésicos e anti-inflamatórios não } \\
\text { esteroidais }\end{array}$ & $\begin{array}{c}1^{\circ} \text { ano }-93,33 \% \\
2^{\circ} \text { ano }-98,44 \\
3^{\circ} \text { ano }-98,61 \% \\
4^{\circ} \text { ano }-100 \% \\
5^{\circ} \text { ano }-90,00 \% \\
\end{array}$ & $\begin{array}{l}\text { Dor de cabeça, gripe, febre, dor de } \\
\text { garganta, enjoo, dor muscular }\end{array}$ \\
\hline
\end{tabular}


O resultado obtido neste estudo corrobora com a revisão feita por Fernandes e Cebranelli (2015), que colocam os analgésicos e relaxantes musculares como os medicamentos mais utilizados na forma de automedicação no Brasil. A própria ANVISA (2020), no seu $9^{\circ}$ Boletim de Farmacovigilância, afirma o mesmo, apontando a dipirona como medicamento mais consumido no país.

No estudo de Alves et al. (2019), os autores perceberam que a grande maioria dos pesquisados (95,0\%) reconheceu a existência dos danos à saúde devido a automedicação, mesmo assim 99\% faziam uso da automedicação. Segundo Martinez et al. (2014), os estudantes e os profissionais da área da saúde teoricamente conhecem os medicamentos e seus riscos, portanto deveriam evitar a automedicação.

No entanto, Moraes et al. (2018), Carneiro et al. (2019), Pilger et al. (2016), Fonseca et al. (2010), ao compararem estudantes no início da faculdade com os de anos mais avançados do curso, constataram que estar mais próximo ao término do curso se correlaciona positivamente com automedicação e isso pode estar diretamente relacionado com a suposição de que com aquisição gradual de conhecimento ao longo da formação e maior experiência, os acadêmicos tornam-se mais confiantes para se automedicar.

Pilger et al. (2016), identificaram que alunos da instituição pública se automedicam mais do que os alunos da instituição particular, com 94\% e 86\%, respectivamente. Quanto à distribuição dos alunos conforme o período que cursavam a prevalência de automedicação foi menor entre os alunos do $1^{\circ}$ ano (79\%) e maior entre os alunos do $4^{\circ}$ ano (99\%). Os autores ainda destacaram que a maior parte dos participantes tinha a faixa de idade de 22 a 24 anos (35\%), sendo que a faixa dos 16 aos 19 anos teve a menor prevalência de automedicação (80\%), enquanto alunos de 22 até 39 anos tiveram a prevalência mais alta (94\%). Silva et al. (2014), identificaram dados semelhantes quanto a faixa etária, em que alunos do quarto semestre entre a faixa etária de 21 e 25 anos tiveram $100 \%$ de automedicação, enquanto que os do primeiro semestre na média de 20 anos corresponderam a $86,5 \%$ de automedicação.

Em todos os estudos analisados, observou-se a prevalência do sexo feminino entre estudantes do curso de Enfermagem e Medicina, e ainda que as mulheres são as que mais se automedicam. De acordo com Souza et al. (2011). o curso de Enfermagem é representado majoritariamente por mulheres. Esse fator pode inferir uma autolimitação dos estudos para a prevalência de automedicação entre as mulheres.

No entanto, no Brasil, estudos apontaram as mulheres como mais representativas em relação à prática de automedicação (Souza et al., 2011). Corroborando com esse contexto, Santos et al. (2019), em seu estudo para avaliar a ocorrência de cefaleia entre alunos de medicina, evidenciou uma maior susceptibilidade das mulheres à cefaleia, e consequentemente a automedicação.

No estudo feito por Souza et al. (2011) para verificar o alívio da dor em acadêmicos de Enfermagem, foi constatado que 96,4\% do sexo feminino faziam automedicação. Pilger et al. (2016) constataram que a automedicação foi referida por $88 \%$ dos homens e 93\% das mulheres. Quanto a isso, Martinez et al. (2014) enfatizam que a exposição do gênero feminino no uso da automedicação vem desde a infância até vida adulta, na procura frequente dos serviços de saúde através de programas e campanhas que são direcionadas ao público feminino, de certo modo, influenciando no autocuidado e por conseguinte na realização da automedição.

Dentre os motivos em geral que os alunos referem como causas da automedicação estão o alívio de dores em geral de forma imediata, falta de tempo para ir ao médico, já ter passado em consulta pelo mesmo motivo então já sabe como se medicar, demora no agendamento de consulta médica, o aluno acredita que possui conhecimento científico adequado para se automedicar, facilidade de acesso, e outros conforme apresentado na tabela 2.

O levantamento feito por Gama e Secoli (2017) descreve como motivos da automedicação a experiência prévia com os sintomas e a doença, a crença de que se conhece a doença, limitação financeira para o cuidado com a saúde e ainda, a atitude do doente frente à doença. Há ainda o hábito de se manter nos domicílios, medicamentos que não foram utilizados pelo não uso da 
quantidade prescrita ou pela aquisição de caixas com quantidade maior, podendo justificar o aparecimento de antibióticos na relação dos medicamentos utilizados para automedicação (Silva et al, 2014).

Martinez et al. (2014) afirmam que o grau de conhecimento sobre o assunto pode gerar uma consciência do risco de se automedicar, mas, por outro lado, pode causar uma falsa segurança no uso desses medicamentos, já que o acesso a essa informação e maior. Embora os estudantes da área da saúde tenham maiores conhecimentos, sabe-se que mesmo profissionais experientes tem cautela no uso e na prescrição dessas medicações (Martinez et al., 2014).

A prática de automedicação, segundo os estudantes, é influenciada por parentes e amigos, por meio da leitura de bulas, conversa com profissionais de saúde, uso de prescrições anteriores, conhecimento sobre medicamentos utilizados previamente e propagandas em mídias (televisão/rádio/internet) (Gama \& Secoli, 2017). No estudo de Silva et al. (2012), a orientação de um parente ou amigo ou o uso da medicação em tratamentos anteriores, prevaleceu como determinante para realização de automedicação no quadro atual, com $41,2 \%$ e $39,6 \%$, respectivamente.

Quanto ao local de aquisição dos medicamentos utilizados na automedicação, Pilger et al. (2016), Gama \& Secoli (2017) e Silva et al. (2012), afirmaram que é diretamente em farmácias. Gama e Secoli (2017) ainda relatam sobre o fácil acesso devido ao armazenamento na própria residência ou de parentes/amigos/vizinhos e ainda, os alunos terem adquirido durante estágios em Unidades Básicas de Saúde (UBS) ou hospital. Silva et al. (2012), mencionam o uso de amostras grátis de medicamentos como um dos meios de praticar a automedicação. Pilger et al. (2016) ainda referem uma forma menos comum de acesso, a partir da compra com prescrição antiga. A reutilização de receitas médicas antigas pode ser vista como um grande problema relacionado à automedicação visto que, muitas vezes, um indivíduo pode apresentar sinais e sintomas semelhantes aos verificados durante o período em que fez uso de determinada medicação (Tognoli et al., 2019).

Um aspecto que pode reforçar o hábito da automedicação pode ser a baixa frequência de efeitos adversos observada pelos sujeitos estudados, principalmente se tratando de analgésicos (Martinez et al., 2014). Os analgésicos e antitérmicos classificados como medicamentos atuantes no sistema nervoso, são fármacos de venda livre (over the counter), e podem representar o autocuidado dos pacientes (Silva et al., 2012). Entretanto, como qualquer medicamento, é capaz de produzir reações adversas, mascarando quadro clínico de maior gravidade. Silva et al. (2012), identificaram a prevalência de reações adversas a medicamentos, pelo menos uma vez na vida, foi de $21,3 \%$.

Efeitos adversos graves podem ocorrer a depender da classe medicamentosa em uso, como é o caso dos moderadores de apetite cuja a prevalência de reações adversas é significante, conforme destacado no estudo de Zubaran \& Lazzaretti (2013). Os autores descreveram que cerca de 10 a $20 \%$ dos voluntários suspenderam o uso desses agentes devido aos efeitos adversos, como irritabilidade, nervosismo, insônia, tristeza, sinais e sintomas físicos emergenciais.

Em contraponto, Souza et al. (2011), ressaltam que, mesmo que os medicamentos utilizados pelos estudantes sejam isentos de prescrição, o consumo irracional dessas drogas pode causar efeitos adversos desastrosos. Os autores destacam ainda que, o tratamento paliativo da dor pode retardar a elucidação do diagnóstico e o tratamento adequado, contribuindo para a cronificação da experiência dolorosa.

A respeito disso, pode-se mencionar a cefaleia por abuso de analgésicos, definida como uma cefaleia diária ou quase diária, com duração maior que quatro horas por dia durante um período maior que quinze dias em um mês (Santos et al., 2019). Portanto, é consenso na literatura, que o primeiro passo é a parada imediata do uso de medicações analgésicas (Santos et al., 2019). Carneiro et al. (2019), afirmam que a alta taxa de automedicação em indivíduos com cefaleia, pode atrapalhar a sua prevenção, devido ao fato de a maioria dos acadêmicos tratarem somente as crises da doença.

Se tratando de universitários, e sabendo da prática de consumo de bebida alcoólica e outras drogas ilícitas nesse meio, Moraes et al. (2018) constatou o uso irregular de fármacos com álcool e/ou tabaco e/ou outras drogas ilícitas em 28,32\% dos participantes. Os autores ressaltam o risco de tal prática, visto que estas substâncias podem alterar as propriedades 
farmacocinéticas e farmacodinâmicas dos medicamentos e, como consequências, suas propriedades terapêuticas, aumentando o risco da toxicidade deles.

Santos et al. (2012), identificaram que além de se automedicarem, 43,82\% dos estudantes de enfermagem, costumam indicar medicamentos a outras pessoas. Moraes et al. (2018), afirmam que este é um fato preocupante, pois pode ser fator para perpetuação da prática da automedicação. Além disso, Santos et al. (2012), destacam que esta é uma prática ilegal de acordo com o Código de Ética de Enfermagem, segundo Art. 48 o enfermeiro não pode prescrever medicamentos exceto os previstos em legislação vigente e em caso de emergência e pelo Art. 30 administrar medicamentos sem conhecer a ação da droga e sem certificar-se da possibilidade dos riscos motivo pelo qual o graduando faz uso da automedicação é o conhecimento da patologia, ação da droga e alívio rápido dos sintomas apresentados.

Assim, a automedicação torna-se um problema de grande proporção com várias questões inseridas e aparentemente como uma prática de difícil controle (Santos et al., 2012). Foi constatado, que no caso dos estudantes de medicina e enfermagem, a automedicação é influenciada pela progressão do acadêmico ao longo do curso e que, embora tenha malefícios reconhecidos, a automedicação desenvolve um papel importante ao colaborar para o descongestionamento do sistema público de saúde, auxiliando na resolução de problemas simples, que não requerem o acompanhamento médico (Pilger et al., 2016).

Entende-se que a automedicação não é uma prática que deva ser erradicada, mas, sim, bem orientada, e que, para que isso aconteça, é necessária uma mudança nos paradigmas do ensino médico, o qual corresponde à base do problema (Pilger et al., 2016).

Por fim, Gama e Secoli, 2017 e Tognoli et al. (2019), indicam como estratégias a implementação de proposta pedagógica educativa sobre automedicação em grade curricular de cursos de graduação em medicina e enfermagem, com a inserção de tópicos que contemplem a discussão sobre o uso racional de medicamentos, alertando os estudantes sobre os limites e responsabilidades de suas ações, sobretudo em relação a indicação de medicamentos e ao gerenciamento responsável dos medicamentos afim, de garantir a segurança do paciente, para reduzir esse ato e capacitar profissionais cada vez mais qualificados.

\section{Considerações Finais}

O estudo evidenciou o uso da prática da automedicação entre os alunos de enfermagem e medicina, com aumento significativo nos períodos finais do curso. O conhecimento adquirido e acumulado ao longo do curso está relacionado com uma maior confiança na prática de automedicação, principalmente entre estudantes do sexo feminino.

Os fármacos mais utilizados na automedicação são os analgésicos/antitérmicos, seguidos de longe pelos antibióticos, antigripais e antialérgicos. Com exceção dos antibióticos, os demais são medicamentos isentos de prescrição, o que facilita a aquisição, mas não impede que o uso incorreto possa causar danos à saúde.

É importante destacar que as instituições de ensino possam planejar ações que visem minimizar essa prática entre os jovens estudantes e futuros profissionais. Abordagens do tema em disciplinas da graduação, programas educativos que possam proporcionar a esses estudantes mais conhecimento sobre os risco e efeitos associados à prática de automedicação se realizada de forma indiscriminada podem ser estratégias interessantes.

Como limitação desse estudo, menciona-se a não inclusão de artigos em outras línguas, o que reduziu a quantidade de produções inseridas nessa revisão, além de serem incluídos somente pesquisas realizadas com alunos dos cursos de enfermagem e medicina. Recomenda-se a realização de novos estudos sobre o tema, avaliando de forma mais profunda o conhecimento desses alunos sobre os medicamentos que utilizam, sobre suas farmacocinéticas, farmacodinâmicas, interações medicamentosas e quais seus efeitos adversos, uma vez que foi detectado que ao mesmo tempo em que eles dizem saber dos riscos, continuam 
fazendo o uso da automedicação indiscriminadamente. Isso supõe que o conhecimento sobre as drogas usadas ainda esteja aquém do necessário.

A automedicação não é uma prática que deva ser eliminada, mas utilizada com cautela e orientações corretas, entretanto, é necessário modificar os paradigmas na formação acadêmica para a formação de profissionais mais qualificados visando a segurança do paciente.

\section{Referências}

Alves, D. R. F., Abrantes, G. G. D., Martins, H. K. A., Lima, A. M. C. L., Ramos, F. F. V. R., Santos, A. C. M. D., ... \& Ribeiro, G. D. S. (2019). Automedicação: prática entre graduandos de enfermagem. Revista enferm UFPE, Recife, 13(1), 363-70.

Andrade, S. M., Cunha, M. A., Holanda, E. C., Coutinho, G. S. L., Verde, R. M. C. L., \& de Oliveira, E. H. (2020). Caracterização do perfil das intoxicações medicamentosas por automedicação no Brasil, durante o período de 2010 a 2017. Research, Society and Development, 9(7), e236973952-e236973952.

Anjos, S., I. \& Oliveira, A. H. G. (2020). A história dos medicamentos e o uso das fórmulas: a conscientização do uso adequado. Revista JRG de Estudos Acadêmicos, 3(7), 475-488.

Arrais, P. S. D., Fernandes, M. E. P., Pizzol, T. S. D., Ramos, L. R. M. S., Serrate, L. V. L., Tavares, N. U. L., Farias, M. R., Oliveira, M. A. \& Bertoldi, A. D. (2016). Prevalência da automedicação no Brasil e fatores associados. Revista de Saúde Pública, 50(Suppl. 2), 13s. https://doi.org/10.1590/s15188787.2016050006117

Baggio, M. A., \& Formaggio, F. M. (2009). Automedicação: Desvelando o descuidado de si dos profissionais de enfermagem. Rev. enferm.UERJ, Rio de Janeiro, 17(2), 224-228.

Bermudez, J. A. Z., Esher, A., Osorio-de-Castro, C. G. S., Vasconcelos, D. M. M. D., Chaves, G. C., Oliveira, M. A. \& Luiza, V. L. (2018). Assistência Farmacêutica nos 30 anos do SUS na perspectiva da integralidade. Ciência \& Saúde Coletiva, 23, 1937-1949.

Behzadifar, M., Behzadifar, M., Aryankhesal, A., Ravaghi, H., Baradaran, H. R., Sajadi, H. S., Khaksarian, M. \& Bragazzi, N. L. (2020). Prevalence of selfmedication in university students: systematic review and meta-analysis. Eastern Mediterranean Health Journal. 26(7), 846- 852.

Bispo, N. S., Ferreira, M. G., Vasconcelos, A. C., \& Esteves, M. B. (2018). Automedicação: solução ou problema? Seminário Estudantil de Produção Acadêmica, 16.

Botelho, L. L. R., Cunha, C. C. A., \& Macedo, M. (2011). O método da revisão integrativa nos estudos organizacionais. Gestão e Sociedade. 5(11), 121-136. https://doi.org/10.21171/ges.v5i11.1220

Brasil. Agência Nacional de Vigilância Sanitária. (2011). Consulta Pública $\mathrm{n}^{\circ} \quad 95$, de $19 \quad$ de novembro de 2001. http://www4.anvisa.gov.br/base/visadoc/CP/CP\%5B2735-1-0\%5D.PDF.

Brasil. Agência Nacional de Vigilância Sanitária. $9^{\circ}$ Boletim, Brasília. Disponível em: https://www.gov.br/anvisa/pt-br/assuntos/noticias-anvisa/2020/divulgado9-boletim-de farmacovigilância>

Brasil. Ministério da saúde. Biblioteca Virtual em Saúde. (2012). http://bvsms.saude.gov.br/bvs/dicas/255_automedicacao.html \#: :text=O\%20uso\%20de\%20rem\%C3\%A9dios\%20de,intoxica\%C3\%A7\%C3\%A3o\%20e\%20resist\%C3\%AAncia\%20aos\%20rem\%C3\% .

Brasil. Ministério da Saúde Secretaria de Atenção à Saúde. Portaria SAS/MS No 3916, DE 30 DE OUTUBRO DE 1998.

Brasil. Ministério da Saúde Secretaria de Atenção à Saúde. Portaria SAS/MS Nº 834, DE 14 DE SETEMBRO DE 2013.

Carneiro, A. F., Neto, P. G. C., Ferreira, J. F. I. S., Garcia, B. F., Silva, F. D. A. C., \& Leal, P. R. L. (2019). A prevalência de cefaleia e fatores psicossociais associados em estudantes de medicina no Ceará. Revista de Medicina, 98(3), 168-179.

Cavalcante, C. S., \& Khouri, A. G. (2019). Atenção farmacêutica nas intoxicações por automedicação. Referências em Saúde da Faculdade Estácio de Sá de Goiás-RRS-FESGO, 2(1).

Domingues, M. P. S., Brandt, G. P., Oliveira, A. P. R., Souza, S. J. P., Ramires, M. A., \& Burci, L. M. (2017). Automedicação entre os acadêmicos da área de saúde. Visão Acadêmica, 18(2).

Silva, F. M., Goulart, F. C., \& Lazarini, C. A. (2014). Caracterização da prática de automedicação e fatores associados entre universitários do curso de Enfermagem. Revista Eletrônica de Enfermagem, 16(3), 644-51.

Moraes, L. G. M., Bernardina, L. S. D., Andriato, L. C., Dalvi, L. R., \& de Sousa Loyola, Y. C. (2018). Automedicação em acadêmicos de Medicina. Revista da Sociedade Brasileira de Clínica Médica, 16(3), 167-170.

Tognoli, T.A, de Oliveira Tavares, V., Ramos, A. P. D., Batigalia, F., de Godoy, J. M. P., \& Ramos, R. R. (2019). Automedicação entre acadêmicos de medicina de Fernandópolis-São Paulo. Journal of Health \& Biological Sciences, 7(4), 382-386.

Fernandes, W. S.; \& Cembranelli, J. C. (2015). Self Medication and irrational use of medications: role of professional pharmacist to combat this pratice. Revista Univap. 21(37). 
Fonseca, F. I. R. M. D., Dedivitis, R. A., Smokou, A., Lascane, E., Cavalheiro, R. A., Ribeiro, E. F., \& Santos, E. B. D. (2010). Frequência de automedicação entre acadêmicos de faculdade de medicina. Diagn. tratamento.

Gama M. S. A., \& Secoli. R. S. (2017). Automedicação em estudantes de enfermagem do estado de Amazonas/Brasil. RGE. Revista Gaúcha de Enfermagem. Mar;38(1): e 65111;2017.

Macedo, G. R., do Carmo, B. B., Castro, G. F. P., \& Correa, J. B. (2016). O poder do marketing no consumo excessivo de medicamentos no Brasil. Revista Transformar, $\mathrm{X}$ ed.

Martinez, J. E., Pereira, G. A. F., Ribeiro, L. G. M., Nunes, R., Ilias, D., \& Navarro, L. G. M. (2014). Estudo da automedicação para dor musculoesquelética entre estudantes dos cursos de enfermagem e medicina da Pontifícia Universidade Católica-São Paulo. Revista Brasileira de Reumatologia, 54(2), 90-94.

Melo, R. C., \& Pauferro, M. R. V. (2020). Educação em saúde para a promoção do uso racional de medicamentos e as contribuições do farmacêutico neste contexto. Brazilian Journal of Development, 6(5), 32162-32173.

Moher, D., Liberati, A., Tetzlaff, J., \& Altman, D. G. (2015). Principais itens para relatar revisões sistemáticas e meta-análises: a recomendação Prisma. Epidemiol Serv Saúde. 24(2):335-42. DOI: 10.5123/s1679-49742015000200017

Mota, D. M., Silva, M. G. C. D., Sudo, E. C., \& Ortún, V. (2008). Uso racional de medicamentos: uma abordagem econômica para tomada de decisões. Ciência \& Saúde Coletiva, 13, 589-601.

Naves, J. O. S., Castro, L. L. C., Carvalho, C. M. S., \& Hamann, E. M. (2010). Automedicação: uma abordagem qualitativa de suas motivações. Ciência e Saúde Coletiva. 15(1): 1751-1762, 2010.

Pilger, M. C., Dombrowski, G., Rebelo, M., \& Tomasi, E. (2016). Automedicação entre acadêmicos de Medicina das Universidades Católica e Federal de Pelotas/RS. Rev. AMRIGS, 26-31.

Santos, B. D., Souza, L. G. D., Delgado, N. M., \& Torres, W. O. (2012). Incidência da automedicação em graduandos de Enfermagem. J. Health Sci. Inst.

Santos, R. D., Rêgo, R. C. D. S., Santos, V. L. B., \& Prado, M. R. (2019). Prevalência de cefaleia e seus impactos em estudantes de medicina em uma universidade pública. Rev. bras. neurol, 5-8.

Santos, R., Neto, A., Dantas, C., Cutrim, C., Sales, R., Silva, M., Próspero, D., \& Nunes, N. (2019). A influência da internet no processo de automedicação: uma revisão integrativa. Brazilian Journal of Health Review. 2. 4310-4323. 10.34119/bjhrv2n5-034.

Schuelter-Trevisol, F., Trevisol, D. J., Jung, G. S., \& Jacobowski, B. (2011). Automedicação em universitários. Rev. Bras. Clin. Med, 9(6), $414-17$.

Souza, L. A. F., Silva, C. D. D., Ferraz, G. C., Sousa, F. A. E. F., \& Pereira, L. V. (2011). Prevalencia y caracterización de la práctica de automedicación para alivio del dolor entre estudiantes universitarios de enfermería. Revista Latino-americana de enfermagem, 19(2), $245-251$.

World Health Organization (WHO). (1997) Internacional farmacêutica federation. The role of the pharmacist i the fight against the HIV-AIDs pandemic. A joint declaration between the WHO and IPF.

World Health Organization. (1998). The role of the pharmacist in self-care and self-medication. Report of the 4th WHO Consultive Group on the role of the pharmacist. The Hague: World Health Organization.

Zubaran, C., \& Lazzaretti, R. (2013). Uso de moderadores de apetite entre estudantes da área da saúde na Região Sul do Brasil. Einstein (São Paulo), 11(1), 4752 . 